\title{
Mobilités géographiques et écarts de pouvoir au sein de trois entreprises mondialisées. Mobiles, immobiles et « ubiquistes »
}

Geographic Mobility and Power Differentials in three Multinationals. Mobile, Immobile and "Ubiquitous" Persons

Movilidades geográficas y diferenciales de poder en tres empresas

multinacionales. Móviles, inmóviles y "ubiquistas"

Laura Gherardi et Philippe Pierre

\section{(2) OpenEdition}

\section{Journals}

Édition électronique

URL : https://journals.openedition.org/remi/5050

DOI : $10.4000 /$ remi.5050

ISSN : $1777-5418$

Éditeur

Université de Poitiers

Édition imprimée

Date de publication : 1 février 2010

Pagination : 161-185

ISBN : 978-2-911627-54-5

ISSN : 0765-0752

\section{Référence électronique}

Laura Gherardi et Philippe Pierre, « Mobilités géographiques et écarts de pouvoir au sein de trois entreprises mondialisées. Mobiles, immobiles et « ubiquistes » », Revue européenne des migrations internationales [En ligne], vol. 26 - nº | 2010, mis en ligne le 01 février 2013, consulté le 16 avril 2022. URL : http://journals.openedition.org/remi/5050 ; DOI : https://doi.org/10.4000/remi.5050 


\title{
Mobilités géographiques et écarts de pouvoir au sein de trois entreprises mondialisées Mobiles, immobiles et « ubiquistes »
}

\section{Laura GHERARDI* et Philippe PIERRE**}

\author{
"C'est l'une des forces des dominants d'avancer sous le \\ masque de l'individualisme et du mérite personnel tout en \\ pratiquant dans la discrétion le collectivisme, le souci de \\ l'héritage et de la transmission des avantages acquis. " \\ (Pinçon et Pinçon-Charlot, 2007 : 264)
}

Si les siècles précédents ont fait naître, en France, des mobilités d'apprentissage où la jeunesse la plus argentée était envoyée dans des comptoirs à l'étranger, dans le circuit des familles amies, alliées ou clientes (Wagner, 2007 : 15), puis des mobilités professionnelles inscrites en lien étroit avec l'État et sa politique industrielle dans les anciennes colonies notamment (Boltanski, 1982), notre époque semble marquée par la mobilité géographique de classes sociales moyennes et supérieures salariées liées à la gestion des affaires et des entreprises (Lazarova et Taylor, 2008). La mobilité physique n'y est en rien étrangère à l'expérience des dominants. Les voyages de longues distances sont majoritairement un privilège des résidents en Europe occidentale et du Sud ainsi que des États-Unis, et les $20 \%$ les plus riches d'entre eux voyagent 3,5 fois plus loin que les $20 \%$ plus pauvres (Doyle et Nathan, 2001).

Ainsi, si le processus de mondialisation peut s'entrevoir comme un espace d'échelle mondiale qui devient pertinent comme espace social, en plus de déjà faire sens comme environnement naturel, c'est-à-dire comme rapport social aux réalités

* Docteur de Recherche en Sciences Sociales (U.C. Milan - E.H.E.S.S. Paris). Actuellement en programme post-doctoral (Visiting Fellow au sein de la Faculté de Sociologie à la London School of Economics). Chercheur associé à la Faculté de Sociologie de l'Université Cattolica de Milan et membre du Laboratoire ARC, laura.gherardi@unicatt.it

** Sociologue. Consultant. Ancien DRH en entreprise. Directeur du cycle « Diversité et Management interculturel » de la formation continue de Sciences-po Paris, philippe.pierre22@wanadoo.fr 
biologiques et physiques (Lévy, 2008 : 11), nous essaierons de montrer, dans cet article, que la distance parcourue par un individu est un indice pauvre du rapport à son inscription professionnelle dans un espace social mondial. Quand sommes-nous, du reste, vraiment «mobiles » en nous déplaçant en entreprise quand il paraît possible, pour certains, de s'y déplacer toujours dans un même monde social, celui de son groupe d'appartenance, et, par accumulation, d'y obtenir des ressources sociales qui permettent de faire valoir avantages et privilèges sans être transformés ?

En France, ce sont les classes supérieures qui contribuent le plus fortement à l'augmentation des effectifs des actifs français étrangers. Cet article interrogera en quoi la mobilité géographique internationale, définie ici comme déplacement professionnel dans un espace physique mondialisé, dote des individus de ressources en vue d'occuper des positions au sein de l'espace de concurrence qui est celui de l'entreprise et en quoi cette mobilité doit être reliée, en cet espace, au pouvoir d'influence sur la mobilité et/ou sur l'immobilité d'autres individus concurrents. Est-ce, pour les cadres et dirigeants en entreprise, l'accès à la mobilité internationale, en elle-même, qui hiérarchise certains groupes sociaux, ou bien la valeur sociale attribuée à ces expériences ou bien encore les réseaux dans lesquels, en se déplaçant, les individus peuvent s'insérer et acquérir davantage encore de pouvoir ?

Les réflexions sociologiques sur l'émergence d'une supposée nouvelle élite transnationale se passent souvent de données empiriques et notamment de données détaillées sur les mobilités des groupes sociaux du haut de l'échelle sociale ${ }^{1}$. Les raisons le plus souvent invoquées de ce désintérêt tiennent à la méfiance des grandes entreprises vis-à-vis de l'apport des sciences sociales, certainement aussi à la faiblesse des allocations de recherche ayant pour objet les couches sociales privilégiées, à la difficulté de repérage des interviewés, à leur disponibilité réduite face à la durée nécessaire des rencontres, mais également à ce que Pinçon et Pinçon-Charlot (2007) appellent la «timidité sociale » du chercheur qui fait que « l'autocensure est souvent la règle, le chercheur choisissant de travailler à partir de déclarations publiques de dirigeants ou de patrons plutôt que de solliciter un entretien ». De plus, en sociologie tout

1 A contrario, une abondante littérature sociologique existe sur les individus situés en bas de l'échelle sociale, constatant des pratiques de mobilité fort différentes (en termes de territoires, d'identités culturelles, de modes d'intégration sociale et institutionnelle...) pour l'étranger, pour l'immigrant qui se déplace pour rester, le migrant qui est en transit, vient seulement pour travailler, traverse les territoires, parfois sous la couverture d'un visa touristique pour faire le « commerce de valise », retourne à la maison ou repart ailleurs, pour le nomade qui, par son mouvement, assure la cohérence de sa culture et de son groupe qui se déplace avec lui, pour le touriste qui voyage en symbole de l'évasion, pour le "vagabond » qui éprouve le monde comme insupportablement inhospitalier et qui erre, poussé par la nécessité, pour le sanspapier, pour l'ouvrier des pays du Sud qui vit une mondialisation par le «bas »... «Le déraciné, en tant que figure paradigmatique du monde migrant, s'éloigne et fait place à une autre figure, encore mal définie mais dont on sait qu'elle correspond à celle d'un migrant qui se déplace et fait appel à des alliances à l'extérieur de son groupe d'appartenance, sans pour autant se détacher de son réseau social d'origine » (Diminescu, $2009: 275$ ). 
comme en histoire, les sujets des recherches traitent très majoritairement de questions à l'échelle nationale. C'est peut-être parce que les études sur la mobilité géographique dépendent, en entreprise, d'un grand nombre de variables (liées au développement stratégique de la firme, aux choix d'investissements, aux types d'activité et de technologies, aux types de gestion des ressources humaines, aux motivations des cadres à partir, à la nature des contrats de travail et des politiques d'accompagnement...) que l'on hésite sur l'approche théorique à privilégier (sociologie des migrations, théorie des organisations, économie internationale, gestion des ressources humaines...) et sur les questions à traiter. D'autant que toutes les modalités de déplacement à l'échelle locale existent dorénavant, à l'échelle mondiale, en entreprise : migrations, navettes quotidiennes, déplacements pendulaires, circulations de travail, missions transfrontalières (Lévy, 2008).

L'inexistence affichée de problèmes sociaux et humains par nombre de « migrants de luxe », leur absence de militantisme syndical, contribuent certainement également à les rendre peu visibles mais puissants. C'est même, à notre sens, cette absence de données de terrain qui fonde certains aspects rhétoriques de la littérature consacrée à la mobilité internationale d'un personnel de haut degré de qualification, détenant le monopole de la construction d'un imaginaire en la matière et qui fait systématiquement l'éloge de la mobilité des effectifs, en toutes ses formes, au lieu de peser ressources et coûts humains induits, prix et épreuves.

On peut ainsi dire que l'on a du mal à rendre compte des comportements de déplacements de ces salariés dans l'espace des entreprises mondialisées autrement que par des logiques collectives massifiées en les noyant dans une « internationale des cadres ». L'asymétrie des positions est souvent réduite aux inégalités de déplacements physiques, de revenus, de consommations. À première vue, ce qui tiendrait ensemble cette classe «mobile » semblerait être davantage une même perception du monde et des pratiques similaires de déplacement. Mais ceci revient souvent à passer sous silence les niveaux de pouvoir et de contraintes dans l'univers du travail, aboutit à nier le fait que les rapports de domination sont aussi des rapports de sens qui sont lentement perçus comme légitimes pour ceux qui en profitent. Ce sont certainement de tels oublis qui font que subsiste l'idée d'une élite homogène composée de « citoyens du monde » qui se reconnaîtraient en un regard et agiraient de concert. Face aux idées largement soutenues par la littérature managériale la plus diffusée, Wagner (1998), tout en nommant ces classes sociales montantes « les nouvelles élites de la globalisation », a su opposer un « internationalisme des managers » (Wagner, 2007 : 50) à un cosmopolitisme propre aux membres de l'aristocratie de l'argent, tels que les définissent aussi Pinçon et Pinçon-Charlot (2006). Cette aristocratie de l'argent mobilise précisément un ensemble de ressources liées à une appartenance à un groupe qui assure des liaisons internationales permanentes et utiles, matérialisées dans des clubs sélects, des rallyes, des cercles, des réceptions... Le « grand bourgeois » est au-dessus de la définition par la profession. Les logiques distinctives incorporées dans les pratiques et la perception de soi, son essence « grande bourgeoise » en quelque sorte, précède son existence sociale et son inscription dans le monde du travail (Pinçon et Pinçon-Charlot, 2006). 
Dans une précédente étude de terrain réalisée dans trois multinationales, nous avions contesté l'architecture de croyances selon laquelle les cadres, à une échelle mondialisée, partageaient une même expérience de mobilité réussie et avions montré qu'en réalité, le siège de la direction des trois multinationales étudiées se posait à l'entrecroisement entre des formes de mobilités différentes que nous avions appelés configurations "spatio-temporelles ». Chacune d'entre elles, par sa direction et sa durée, comportait une articulation différente des coûts humains à supporter pour les expatriés, les impatriés, les cadres internationaux et les dirigeants alors distingués ${ }^{2}$. Si les systèmes de transport et de télécommunication confèrent des potentiels de vitesse considérables pour des mobilités de plus en plus réversibles, pour ceux qui se déplacent, la mobilité y apparaissait comme un impératif de carrière, une épreuve touchant la sphère personnelle et familiale et débouchait aussi sur une diversité de constructions identitaires ${ }^{3}$, contredisant ainsi l'idée d'une élite unie sur le plan de la socialisation et des représentations professionnelles.

L'amélioration continue des moyens de transport motorisés et l'accroissement de l'usage des TIC font que les cadres et dirigeants profitent à la fois d'une nouvelle « connexité » (c'est-à-dire le franchissement des distances spatiales par un moyen technique) et d'une nouvelle « contiguïté » ${ }^{4}$ à mieux comprendre. Précisément, dans cet article, nous voulons détailler les écarts de pouvoir qui subsistent entre cadres intermédiaires, cadres supérieurs et dirigeants d'une même multinationale par l'étude, d'une part, des logiques d'actions que cachent les utilisations des moyens de déplacement au quotidien et la mesure, d'autre part, des effets de ces déplacements, en termes d'inégalités. Ceci va nous conduire au repérage d'un mécanisme, répandu dans la division actuelle du travail, de maintien et d'accumulation de relations sociales - de la part de ceux que nous nommons ici « ubiquistes », les dominants dans le réseau - au moyen de la mobilité (et de l'immobilité aussi) d'autres personnes.

Les résultats de notre travail d'enquête se basent sur une cinquantaine d'entretiens avec des cadres expatriés, des cadres impatriés et internationaux, des dirigeants (« top management») - appartenant à trois multinationales d'origine française - ainsi

2 Nous avions proposé quatre configurations spatio-temporelles différentes : expatriation d'expertise ou de conquête de marché, mobilité réticulaire à base unique, double mobilité scalène et circulation du top management à mobilité externe (Gherardi et Pierre, 2008).

3 De précédents travaux nous ont amené à conclure que la mobilité internationale incite les cadres mobiles (expatriés ou impatriés) à reconnaître la présence en eux d'un étranger déprécié, aimé ou idéalisé, parfois à reformuler leur sentiment d'appartenance en acte de revendication culturelle. Inscrits ainsi dans des registres d'action multiples et non congruents, dans des situations qui ne sont pas entièrement codées et prévisibles, ces cadres internationaux s'expliquent, se justifient et prennent de la distance par rapport à leurs cercles d'appartenances et d'origines (Pierre, 2003). Cinq stratégies identitaires ont été proposées : celles des « conservateurs », « défensifs », « opportunistes », « transnationaux » et « convertis ».

4 Un mouvement de substitution continu de formes irréversibles de mobilité (migration, mobilité résidentielle...) s'opère vers des formes plus réversibles (mobilité quotidienne, voyage, pendularité...) (Kaufmann et Widmer, 2005). On passe ainsi, dans les pays de la CEE, de 2000 milliards à 4000 milliards de personnes/km par an entre 1970 et 1993. 
que sur un ensemble de données recueillies auprès de grands actionnaires, d'entrepreneurs qui ont construit de grandes fortunes et d'héritiers 5 .

Dans une première partie, cet article s'attachera à peser en quoi un imaginaire «mobilitaire », entendu comme ensemble de significations communes propres à un collectif social, a émergé et s'est diffusé comme caractéristiques souhaitables surplombant la gestion des carrières, la réussite au travail, l'acquisition de compétences et le sens de tout projet professionnel. Nous nous attacherons ainsi au processus de formation d'un imaginaire d'une population mobile pour en comprendre les traits constitutifs. Nous comprendrons mieux pourquoi la mobilité géographique n'est pas un facteur d'homogénéisation d'une élite mais participe, en réalité, d'une distribution inégale de l'autorité légitime derrière une célébration unanime du mouvement et du changement.

Nous verrons, dans une deuxième partie, que certains mobiles vont alors exercer une autorité et d'autres vont y être soumis : une première division s'opère entre les dominants (détenteurs de l'autorité) et les dominés (soumis à l'exercice de cette autorité) qui se structurent selon la défense de leurs intérêts. Nous explorerons les écarts de pouvoir au sein de la population mobile salariée, étudiée en tant que telle et aussi en comparaison de grands actionnaires et membres des élites économiques de vieille date, qui, eux, apparaissent moins assujettis aux rythmes de la production et pour lesquels la mobilité semble plus une option qu'un impératif à satisfaire. Peut-on repérer un capital international qui jouerait comme un «multiplicateur » d'autres formes de capitaux, fonderait de nouvelles oppositions et de nouvelles positions dominantes et ferait de l'échelle mondiale une dimension spatiale légitime pour comprendre des rapports sociaux vus localement (Wagner, 2007 : 5 et 85) ? Nous discuterons aussi de l'hypothèse - non conventionnelle - selon laquelle la mobilité peut être un facteur d'exploitation de certains mobiles par les sédentaires, mais aussi favoriser, dans la division du travail, les «ubiquistes » qui prennent, par délégation et selon les circonstances, figures de mobiles ou d'immobiles.

5 L'enquête repose sur un échantillon de 52 personnes avec la répartition suivante :

- 20 entretiens semi-directifs pour des cadres intermédiaires vivant une mobilité internationale (cadres impatriés en France, cadres expatriés et cadres internationaux) et 7 entretiens pour des membres de la direction (Directeur Général et Seniors Vice-présidents) dans la même firme multinationale, dont le siège est à Paris et que nous appelons « multinationale 1 ».

- 12 entretiens auprès de managers appartenant à deux autres firmes multinationales, de la même taille de chiffre d'affaires que la première, qui ont aussi leurs sièges à Paris, que nous appelons «multinationale 2 » et «multinationale 3 ». Nous distinguons 8 entretiens pour une population appartenant au « middle management » et 4 personnes au « top management ».

Nous avons pu avoir accès à plusieurs plannings de mobilités de cadres et dirigeants rencontrés sur trois années calendaires.

- 8 entretiens auprès d'héritiers de grandes fortunes, dont trois ayant des fonctions opérationnelles dans l'entreprise de famille ;

- 5 entretiens auprès d'entrepreneurs de première génération ayant construit des entreprises de grande taille (dont 3 de nationalité italienne). La durée de ces entretiens a varié d'une à trois heures. À cela s'ajoutent quatre entretiens auprès d'informateurs privilégiés, sur les pratiques de mobilité dans les entreprises, dans les métiers du commerce et du contrôle de gestion. 


\section{L'IMAGINAIRE « MOBILITAIRE » D'UN NOUVEAU TRAVAILLEUR MONDIAL ?}

"Ce sont maintenant les nomades voyageant "léger" et se déplaçant rapidement qui sont aux commandes, mais pas seulement à cause de leur aptitude au délit de fuite." (Bauman, $2003: 23$ )

\section{Diffusion d'un imaginaire « mobilitaire »}

Il y a un imaginaire du mot voyager qui est lié aux pratiques et significations européennes depuis plusieurs siècles à travers les mots d'initiation, errances littéraires, masculines, bourgeoises, scientifiques, héroïques, récréatives (Clifford, 2007)... et prendre la juste mesure du problème revient d'abord à chasser certains mythes, dont la force d'évocation alimente tribunes de journaux et même ouvrages d'experts. Dans le capitalisme post-fordiste, la mobilité géographique intervient précisément comme une nouvelle norme sociale de production, c'est-à-dire qu'elle prend les traits d'une valeur, d'un impératif et d'une épreuve qui façonne des principes de légitimité. Elle est synonyme d'adaptabilité et de flexibilité pour des femmes et des hommes qui président aux destinées des entreprises et doivent se déplacer tout comme la structure carrossée d'un véhicule qui est désormais dessinée en Californie, fabriquée en Italie et assemblée au Brésil avec des composants électroniques conçus dans le New Jersey ou au Japon. Ces nouveaux travailleurs mondiaux - devons-nous les appeler « cosmocrates » (Vertovec et Cohen, 2002) - passent leur temps dans des avions ou des trains rapides que l'on peut prendre à toute heure vers des pays à portée de main, vivent dans les hôtels, fournissent des consultations, exposent leurs idées, contrôlent l'activité des autres, donnent des instructions et signent des accords. Ils publient périodiquement des rapports de gestion, des plans prévisionnels budgétés, des échantillons ou des projections qui, à leur tour, donnent lieu à d'autres réunions.

L'imaginaire « mobilitaire », défini à la fois comme « un univers symbolique objectivé et un espace de capacités d'énonciation et de transformations symboliques » (Barrère et Martuccelli, 2005), qui s'est diffusé largement au cours des trente dernières années, dans une partie de la littérature en sciences humaines et de la littérature managériale internationale, se fonde sur une apologie à trois «têtes » : éloge de la mobilité géographique des personnes liée au flux des investissements productifs et à la nécessité de gérer des dangers plus difficilement quantifiables et prévisibles, éloge de la mobilité au sens plus ample de mobilité des idées favorisant la créativité et éloge de la mobilité comme aptitude de chacun à s'insérer sans cesse dans des réseaux et dans de nouveaux projets. Plusieurs auteurs ont pointé l'existence d'un passage, dans notre société, d'une sédentarité dominante à une « hypermobilité » dominante des élites (Zelinski, 1971 ; Knafou, 2000) et notent, dans le même temps, que le degré d'immatérialité d'un univers social est un signe du prestige social. La valeur d'un manager, d'un dirigeant plus encore, en entreprise, se mesure ainsi au nombre de voyages qu'il effectue chaque mois et, surtout, au nombre de personnes qu'il dirige « à distance ${ }^{6}$.

6 « Comme le suggère Richard Sennett, tout sépare le projet de John. D. Rockfeller de fonder un empire économique et une dynastie familiale par l'enracinement sur un lieu de production à 
L'autre doit être découvert. L'imaginaire « mobilitaire » dit le contraire et célèbre l'occasion à saisir, l'instantanéité, la convivialité, la jeunesse, la transparence, l'égalité (d'accès) et la liberté (de parole) dans une nouvelle " réalité » non hiérarchique, cybernétique, reliant des champs hétérogènes, comme le fait le réseau Internet (Taguieff, 2001). La créativité et l'exigence d'autonomie sont considérés aujourd'hui comme des valeurs en soi et sont des piliers rhétoriques contemporains de la globalisation, de la flexibilisation du marché du travail, et soutiennent un nouveau modèle d'excellence des travailleurs à haut degré de qualification contre des autorités locales fixes et stationnaires. Boltanski et Chiapello (1999) ont su montrer comment un « nouvel esprit du capitalisme » a, en particulier, incorporé la « critique artiste » qui s'était épanouie en mai 1968 en revendiquant "une exigence de libération, d'autonomie et d'authenticité » que le capitalisme hiérarchisé ne pouvait satisfaire dans ses rythmes de production. La valorisation croissante de la mobilité comme ressource, pendant les dernières décennies, a fait que l'exigence de devoir bouger est devenue de plus en plus pressante pour les couches sociales supérieures. Les dominants d'aujourd'hui cultivent donc une culture de lien, dont ils ont hérité et qu'ils entretiennent dans la mobilité. Elle est proposée dans les institutions d'enseignement, dès le plus jeune âge, comme un des critères, plus ou moins explicite, d'appartenance à une élite - pour soutenir la réorganisation d'un capitalisme dont les unités sont dispersées sur l'échiquier mondial. Dans cette perspective, Iyer (2006 : 31) fait état d'un nouveau travailleur mondial qui connaîtrait des « changements que le passager traditionnel sur un paquebot ou un train longue distance n'aurait jamais imaginés ». Et il ajoute : « si tant est qu'il puisse éprouver un lien de parenté avec quelqu'un, ce serait, vraisemblablement, avec les autres membres de l'état du Déracinement » (Iyer, 2006 : 32). Iyer évoque cet être planétaire qui «ne peut pas vraiment se considérer comme un exilé (qui, traditionnellement, laisse derrière lui un pays dès lors perdu), ni un expatrié (qui, en général, a choisi de s'établir à l'étranger pour y vivre) ». «Je ne suis pas vraiment un nomade (qui obéit aux saisons et aux traditions). Et je n'ai jamais été sujet aux violentes perturbations du réfugié : l'homme global se caractérise davantage par le fait de se situer entre les catégories (...)» $(2006: 38)$.

Reich (1993) avait évoqué, en son temps, les « manipulateurs de symboles ». Ces derniers sont d'abord américains mais présents aussi dans d'autres pays où la production de masse fait place à une production «personnalisée ». « Les industries de haute technologie, les activités d'expertise juridique, financière et gestionnaire, la recherche scientifique fondamentale et appliquée, le secteur de l'information et les industries de création forment une avant-garde, avec ses sites et ses vitrines (les « ville monde » comme Londres, New-York, Los Angeles, Paris, Berlin, Tokyo, Shangaï...), sa doctrine organisationnelle (le projet, le réseau, l'équipe, l'implication, le contrôle décentralisé, la responsabilité...) et sa philosophie du travail fondée sur l'individualisation » (Menger, $2002: 6$ ). Ces manipulateurs de symboles travaillent principalement à partir de données, de mots, de représentations, ce qui correspond, aux métiers de chercheurs, aux ingénieurs, avocats, consultants, publicitaires, journalistes,

long terme, et l'attitude d'un Bill Gates qui, inséré dans des réseaux mobiles, ne semble avoir aucune objection au déplacement, voire à défaire ce qu'il a fait dans le passé » (Barrère et Martuccelli, $2005:$ 75). 
promoteurs immobiliers, banquiers, et conseillers de toutes sortes (y compris politiques). Il faut tenir compte également des artistes de cinéma et des musiciens. Le groupe qu'ils forment comprend une large palette, hiérarchisée, d'emplois car certains «tiennent à peine leur rang dans l'économie mondiale », tandis que d'autres «sont tellement demandés dans le monde qu'ils ont du mal à garder trace de tous leurs revenus »(Reich, 1993 : 202). Ces manipulateurs de symboles ont plus souvent des partenaires, des associés, des contacts que des supérieurs hiérarchiques ou des patrons. Bien, voire très bien payés, « ils aiment leur travail », qui consiste à « résoudre des énigmes, mener des expériences, des discussions fréquentes ». Disposant d'une liberté d'action substantielle, ils travaillent en petites équipes dans une entreprise ou un réseau qui fonctionnent en temps réel, ils récoltent le maximum d'informations sur la concurrence et « reçoivent les informations en retour par l'observation rapide des effets de leurs actions sur l'environnement (produits, marchés, réactions des clients) »(de Rosnay, 2004 : XIX). Ils voyagent beaucoup. Reich observe que le discours sur la mobilité internationale comme processus d'accumulation du capital humain qui permettrait d'améliorer le potentiel de recherche et d'innovation et d'assurer ainsi la compétitivité future des économies, n'a cessé de prendre de l'ampleur depuis trente années dans les grandes entreprises et institutions des pays riches (Inkson et Khapova, $2008: 151)^{7}$. La mobilité internationale y apparaît donc, de plus en plus, comme un vecteur d'échange de connaissances et d'enrichissement des individus dans des équipes de travail qui concentrent leurs activités autour de la résolution de problèmes nouveaux posés à l'organisation (équipes dites « cross-fonctionnelle ») ou encore baptisées « virtuelles » (Robbins, 2000) qui n'exigent pas nécessairement le déplacement (coûteux) de la famille. Reich souligne que l'énergie créatrice de ces individus doit être cadrée, « dirigée de manière appropriée vers un fonctionnement utile » : « comme les membres de l'équipe se dispersent après le projet, il importe que soient capitalisés, par l'enregistrement dans des génériques personnels, les apports et les acquis de chacun, même si l'information sur la valeur exacte de chaque contribution est, pour le meilleur ou pour le pire, mêlée à l'évaluation globale du résultat d'ensemble » (Menger, 2002 : 50).

Les manipulateurs de symboles n'ont pas de parcours de carrière linéaire mais conquièrent une employabilité croissante au fil de leurs projets, de leurs engagements de carrières. Les expériences internationales et inter-fonctionnelles sont vécues comme incontournables (Pierre, 2003). La mobilité est ce qui permet au «talent » de multiplier ses manifestations, ses « expositions », c'est-à-dire les défis successifs qui permettent au salarié d'étalonner et d'exprimer au mieux son potentiel ${ }^{8}$. Une carrière réussie

7 Yvonne McNulty et Phyllis Tharenou (2004) définissent six catégories de cadres mobiles dans les FMN "Expatriates assignees, host country nationals, inpatriates moved to the parent company, third-country nationals, multicountry business travelers, global managers managing whole organizations and majors functions from a home base".

8 La figure du talent n'est pas sans rappeler celle, ancienne, du compagnon qui construit son « chef-d'œuvre » au fil de ses expériences nomades ou mieux, celle du « sublime », ouvrier très qualifié sous le Second Empire, qui peut opter pour une succession d'emplois qui le satisfont et valoriser la détention de compétences transférables entre diverses organisations. C'est la valeur de la fidélité à l'entreprise, à son rôle dans une temporalité longue, qui est remise en cause. Pour le «sublime », l'instabilité de l'emploi n'est pas un problème : c'est la solution écrit Bernard Gazier (2003). 
équivaut à une mobilité ascendante au sein d'un échiquier stratifié de réseaux d'interconnaissance et de collaborations récurrentes, parfois éphémères, en plusieurs pays (Menger, 2002 : 44). La division du travail s'opère par zones d'expertise et appariements sélectifs fondés sur des logiques de réputation. La valeur d'un cadre ou d'un dirigeant se base sur la réussite des projets auxquels il a été associé récemment. Les cabinets de recrutement, les chasseurs de têtes, les intermédiaires de toutes sortes (coachs, consultants en organisation...) aident les décideurs à gérer « les bons appariements, maintenir la cotation des réputations sous une pression concurrentielle permanente en alimentant sans cesse le vivier des candidats à la gloire avec de nouveaux talents (...) » (Menger, 2002 : 45). Menger fait état, lui aussi, d'un modèle de carrière où plus la compétence est élevée, plus l'attachement à une seule entreprise fait question. «Le professionnel incarne bien celui qui détient une expertise demandée et prisée, mais il peut espérer en obtenir un meilleur prix par la concurrence de marché que par l'appartenance à une organisation » (Menger, 2002 : 86). Chacun doit devenir « entrepreneur de sa propre carrière » («portfolio worker ») selon l'expression de Handy (1989), avoir de l'intuition, du flair, de la créativité et peu d'attaches. Émerge une figure du manager créatif (Chiapello, 1998) qui s'esquisse au détriment de celle du vieux cadre bureaucratique. "Loin des représentations romantiques, contestataires ou subversives de l'artiste », écrit Menger, «il faudrait désormais regarder le créateur comme une figure exemplaire du nouveau travailleur, figure à travers laquelle se lisent des transformations aussi décisives que la fragmentation du continent salarial, la poussée des professionnels autonomes, l'amplitude et le ressort des inégalités contemporaines, la mesure et l'évaluation des compétences ou encore l'individualisation des relations d'emploi (...)» (Menger, 2002 : 8). Et la boucle est bouclée quand ce ne sont pas les professionnels qui ressemblent aux artistes mais les artistes qui ressemblent aux professionnels.

\section{Dimensions idéologiques des pratiques de mobilité}

Les formes de compagnonnage des sociétés occidentales, le perfectionnement de nombreux corps de métiers, reposent, depuis des siècles, sur la mobilité géographique comme épreuve intellectuelle et spirituelle. Or, dans l'idéologie managériale qui célèbre cet imaginaire "mobilitaire », la mobilité n'a pas d'effets nocifs sur la vie de cadres toujours prêts à apprendre et à faire face à l'évènement. Être mobile est seulement synonyme d'ouverture d'esprit, de découverte et d'expérience accrue (remarquons que la majorité des grandes entreprises ne s'intéressent que depuis peu, dans le domaine des formations permanentes (devenues « interculturelles ») ou celui de la gestion des carrières (devenues « internationalisées »), aux logiques affectives, communautaires ou familiales qui sous-tendent la mobilité et les schèmes d'action intériorisés). Dans cette idéologie managériale, la mobilité est un fait universel, une variable «mono-logique » qui ne varie qu'en intensité mais peu en complexité pour celui qui la vit. L'évènement est comme programmé et ne suscite pas, en théorie, de surprise au fil de l'accroissement des vitesses de transport et des distances parcourues. Ceci constitue, selon nous, une idéologie oublieuse des dilemmes pratiques que rencontrent les acteurs en cours d'action, les obligeant à questionner les référentiels qu'ils mettent en œuvre ; une idéologie oublieuse de ces identités des populations mobiles « à la fois plurielles et partielles », pour reprendre la belle formule de Rushdie (Iyer, 2006 : 224). 
Cet imaginaire «mobilitaire » a aussi partie liée avec une constante valorisation de la diversité comme propédeutique de l'identité professionnelle. «La variété des postes tenus, des patrons, des collaborateurs, des environnements techniques, des contextes culturels : toutes ces expériences rencontrées à l'occasion du parcours professionnel ont pour effet de tester et de renforcer les compétences génériques, c'est-àdire celles qui seront le plus sollicitées lorsque le « haut potentiel » se retrouvera en position de dirigeant : stabilité émotionnelle et résilience, capacités cognitives générales, qualités relationnelles, etc ${ }^{9}$ écrit Miralles (2007 : 92).

Cette idéologie se fonde aussi, selon nous, sur un «alignement » des sédentaires (ou des débutants) sur le discours des autres membres de la profession (les dominants supposés plus expérimentés et compétents). Cabantous (2008) pour les « gens de mer » ou Courty (1995) pour les routiers, décrivent une logique de construction de l'identité d'un groupe social qui renvoie à un discours inventé par les sédentaires (ceux qui restent à terre ou à la maison), par les « autres » de la profession. S'exerce une sorte de fascination-répulsion des sédentaires vis-à-vis des cadres mobiles qui assument une fonction de «passeurs » et entretiennent l'espoir d'une mobilité « fantasmée ». Ceux qui vivent une « internationalisation sur place » n'auraient plus besoin de partir pour éprouver le mouvement du monde (pensons à une assistante de direction qui est sédentaire mais vit bien réellement, en trois langues différentes, les contraintes de plusieurs fuseaux horaires). Ici, le sens même de la mobilité en entreprise changerait de visage : elle ne serait plus le seul monopole des acteurs qui se déplacent, mais une donnée structurelle du monde moderne du travail en tant que tel, comme intégrée aux processus productifs (Barrère et Martuccelli, 2005 : 65). L'ample utilisation des potentiels de vitesse, de la téléphonie, des ordinateurs portables, sert souvent d'abord à préserver, selon nous, une forme de sédentarité ${ }^{10}$, des processus d'ancrage, et ne fait pas forcément diminuer le nombre des voyages d'affaires. Ainsi, le fait d'être physiquement présent lors d'une réunion à l'étranger constitue encore le signe vivace de l'importance qu'un individu accorde à une rencontre. Il choisit d'arbitrer alors consciemment entre « donner de soi » et se servir d'autres moyens pour communiquer. Ces réunions à l'étranger permettent, au dire des interviewés, de "récolter quelques informations contextuelles insaisissables au moyen des TIC ». Ces réunions à l'étranger illustrent aussi le cumul des positions dominantes qui s'enrichit de flux constants d'échanges en différents lieux (un des directeurs interrogés indique, lors de nos enquêtes, qu'il a "en tête des milliers de lancements de produits et c'est son avantage lorsqu' il est en réunion. Il peut déceler plus vite les erreurs, identifier les causes d'un insuccès et orienter le travail des équipes vers une performance atteignable... »). Ce cumul est lié à des savoir-faire qui se multiplient et se renforcent les

9 Christophe Falcoz (2001 : 14) constate que le label même de cadre « à haut potentiel », loin de désigner un statut, représente un entre-deux identitaire, une trajectoire mobile, puisque le cadre à haut potentiel n'est plus un cadre parmi d'autres (mais il peut le redevenir) et n'est pas encore un dirigeant.

10 "Les nouveaux moyens de déplacement ne sont donc pas le signe d'une "hypermobilité" croissante. C'est au contraire pour préserver notre environnement familier et nos ancrages que nous choisissons maintes manières de nous déplacer vite et loin» (Kaufmann, 2008 : 21). 
uns les autres avec les voyages et missions (« on se constitue », par exemple, «d'autant plus aisément un réseau international qu'on parle les langues et, en retour, la fréquence des interactions avec des locuteurs étrangers est essentielle pour entretenir et développer ses compétences linguistiques » constate Wagner (2007 : 45)).

Pour les plus puissants, ceux que nous nommerons l'aristocratie de l'argent (grands actionnaires, rentiers, administrateurs...), l'important est de faire plusieurs choses à la fois quand ils voyagent, de cultiver une polyvalence liée à une « surcapacité » circulatoire : déplacements touristiques, de loisir, voyages d'affaires, migration résidentielle, circulation pendulaire, pèlerinage, shopping... En tentant de changer de cercles sociaux le moins possible, c'est, en grande partie, pour se faire plaisir, se détendre, se cultiver... que cette aristocratie de l'argent bouge. Lussault évoque, à ce propos, une intéressante et nouvelle « hybridation en acte » des pratiques mobilitaires à l'échelle planétaire ${ }^{11}$ qui garde séparés, comme nous le détaillerons plus loin, les espaces professionnels (espaces cosmopolites) dans lesquels transite l'aristocratie de l'argent et ceux dans lesquels transitent les élites managériales salariées (espaces internationaux et transnationaux).

\section{Dispositifs de mobilité professionnelle comme dispositifs de pouvoir en entreprise}

La référence même au terme d'élite suggère souvent, et à tort, que la position que les sujets occupent dans la hiérarchie professionnelle est le seul indice de leur position sociale tout court, comme si les patrimoines personnels, hérités ou rentes de toutes sortes, qui sont passibles d'être transformés en capital financier pur, ne jouaient aucun rôle dans la morphologie sociale. Le mythe tenant à l'existence d'une élite transnationale fonctionne de même en évacuant les questions d'écarts de pouvoir entre cadres intermédiaires et sphères dirigeantes.

\section{Dispositifs de mobilité professionnelle, capital social et inégalités de ressources}

Il y a bien des manières différentes d'être mobile en entreprise qui, dans une première perspective, découlent des potentiels de vitesse procurés par les systèmes techniques ou du niveau de confort des déplacements réalisés ${ }^{12}$.

11 « Ainsi, un pèlerin est aussi, au moins potentiellement, un touriste et retrouve dans son pèlerinage des habitudes de "navetteur", et/où celles de ses déplacements de loisir ; un migrant profite de technologies et de structures de mobilités qui sont celles des touristes, des hommes d'affaires, des pélerins... Parallèlement, ces pratiques s'ouvrent vers des temps de halte, des stases, dont la durée est très variable, valorisées ou non, recherchées ou subies » (Lussault, 2003).

12 Les conditions de transport se révèlent un indicateur faible que l'on ne peut pas généraliser. Un des directeurs généraux de la multinationale 1 interrogé convenait " qu' il y a deux façons de se déplacer en avion. La façon de tout le monde. Mais cela dépend dans quelle classe on voyage. Est-ce que l'on voyage en business ou est-ce que l'on voyage en first? C'est très 
Nos enquêtes font état d'un petit nombre de personnes mobiles qui n'évoluent ni dans la simultanéité ni dans la successivité mais, plus que d'autres, dans des temps croisés et enchevêtrés, en profitant de la contribution des autres. Pour ces populations, l'intensité de relations sur une courte période est ce qui va permettre de les réactiver ailleurs en un temps court lui aussi. Mobiliser en un temps donné des réseaux de relations sûres et un savoir-faire acquis en différents lieux, et avec différents partenaires, est un avantage professionnel majeur. Il tient à une convertibilité des capitaux sociaux, culturels, symboliques détenus car, comme l'écrit Lévy, " l'intensité et la vitesse des dynamiques dépendent alors moins de la fongibilité réciproque des environnements pris dans leur ensemble que de la capacité des acteurs à activer des éléments d'un environnement qui peuvent servir dans un autre »(2008:355).

Pour mieux comprendre cette capacité de certains individus de multiplier leur présence sur des scènes géographiquement dispersées, nous avons eu recours à la notion de «dispositif de mobilité » ${ }^{13}$ qui, pour nous, a pris sa première forme à partir de l'observation des planning du management supérieur auxquels il nous a été permis d'accéder. Nous en donnons ici un court extrait : "Je vous montre mon planning personnel (...) bon, j'étais à San Francisco et à New York non, c'était qui ? C'était quelqu'un de mon équipe, moi j'étais à Los Angeles en février, ensuite on est partis à Madrid, à Francfort, à Londres. À Londres, c'est des voyages d'une journée, de deux journées ou trois journées maximum. À Londres, j'y vais deux ou trois jours par mois, à Madrid encore, on a été deux jours en Scandinavie, deux jours aux États-Unis, encore en Scandinavie. Là mes équipes étaient en Suisse, je les ai envoyées à ma place comme il y a des jours où je devrais être dans deux ou trois endroits aux coins opposés du monde, je pars au Portugal pendant deux jours, la semaine prochaine c' est Bruxelles et Nycosie, vous voyez que chaque semaine ici il y a des voyages » (Directeur de la communication financière, multinationale 1 , siège de Paris).

Tout comme les autres, ce planning personnel bimestriel inclut, en plus de la mobilité du directeur, une mobilité « emboîtée » sur la sienne, celle d'autres collaborateurs. Et d'abord celle des subordonnés qui composent l'équipe. En cela, la culture du lien (alliant mobilité et connectivité) des dirigeants ne peut fonctionner sans culture de contrôle sur les personnes, les processus (normalisés) et les relations sociales (alliant surveillance des marchés, reporting au travers d'indicateurs de gestion...et traçabilité des décisions prises, des profits réalisés au mois le mois). Cette culture du contrôle évolue au rythme des pratiques graduées de communication :

important la classe dans laquelle on voyage, parce que si t'as huit heures de voyage avec tes genoux sur le menton et un gros à côté tu ne dors pas - mais le top du top c'est de voyager en jet, par exemple le groupe P. a trois jets dans lesquels il déplace les cadres dirigeants. Les vrais hypermobiles, dans les entreprises, ceux qui ont des jobs importants, les hypermobiles puissants, je veux dire, parce qu' il y a aussi des hypermobiles à des niveaux de responsabilité pas forcément élevés. Je pense qu'il faut toujours différencier un peu les deux ou quoi. Moi je prends le jet soit quand je suis très pressé, soit parce que les endroits sont mal desservis par les vols de ligne».

13 La notion de dispositif vient de la sociologie pragmatique. Pour une première connaissance, nous renvoyons à Mohamed Nachi (2006). 
depuis les simples modalités « conversationnelles » où la communication supplée à l'absence, à la télé-présence, aux visioconférences, jusqu'aux modalités « connectées » où les interlocuteurs manifestent une forme de "présence » continue malgré la distance. Une double présence (Sayad, 1999) repose toujours sur une capacité à actualiser en permanence le lien avec l'environnement d'origine, tout en établissant des contacts avec les sociétés des pays et lieux de destination. En bref, le directeur interrogé est mobile autant que ses subordonnés, mais il est aussi et surtout doué du don d'ubiquité. Ce directeur utilise au mieux les installations des aéroports notamment, pour accueillir des réunions de quelques heures et qui rassemblent des décideurs en provenance de plusieurs capitales. Il a recours à l'équipement en modems permettant la communication formalisée sur écran en temps réel. Ces réunions, organisées souvent en quelques jours, jouent un rôle important dans ce que Evans qualifie de « technologie de colle » de l'organisation, fondée sur les transferts de savoir entre entités (filiales nationales, siège mondial et autres unités fonctionnelles ou opérationnelles). Le directeur est partiellement présent au siège pendant qu'il voyage en la personne de sa secrétaire et d'un collaborateur qui en sont comme les « doublures locales », ainsi que les nomment Boltanski et Chiapello (1999) dans une perspective théorique de la mobilité comme facteur d'exploitation. Il a donc la possibilité d'être simultanément ici (au siège), là-bas (le lieu de destination de son déplacement) et ailleurs encore (les lieux où ce directeur envoie les membres de son équipe). Sur la base de quelques-uns des plannings que nous avons pu observer, une organisation en «flottille », autour de la personne du directeur, inclut une ou plusieurs personnes qui le représente à l'étranger (mais aussi dans le territoire national) sous une plus longue durée, selon les secteurs. Ces flottes sont normalement constituées par des sujets dont les compétences peuvent être considérées comme partiellement complémentaires à celles du directeur. Ces flottes multiplient les « yeux » et les « oreilles » du directeur sur les scènes « là-bas » quand la raison du déplacement réside, par exemple, dans la nécessité de saisir des informations de contexte (climat social, par exemple). La confiance est un élément essentiel au bon fonctionnement de la flottille mais rien ne dit qu'elle va mécaniquement s'améliorer à mesure que les contacts formels seront répétés entre les membres.

\section{Coexistence spatiale et conditions de la puissance sociale}

Appréhender la mobilité en termes de dispositifs comme nous le faisons dans cet article amène à prendre en considération des variables contextuelles telles que l'organisation de personnes et de ressources qui viennent en délégation du sujet en mouvement. Faire apparaître ces logiques entrecroisées des déplacements, la disposition des ressources et des personnes qui entoure la mobilité d'un autre sujet, signifie rendre visibles tous ces liens qu'une partie de la littérature managériale, en considérant la mobilité en tant que ligne qui relie deux points éloignés de l'espace géographique, ignore. Le directeur étudié en exemple plus haut nous apparaît maintenant comme un sujet placé dans un dispositif qui lui offre la possibilité d'être simultanément présent sur plusieurs scènes, comme un individu doué d'ubiquité, en plus d'être mobile, grâce à l'appareil organisationnel de collaborateurs, assistants, intermédiaires, ambassadeurs locaux, accompagnateurs, représentants... Cette inégalité fondamentale entre le 
directeur et les membres de son équipe, aussi bien qu'entre le management supérieur et le management intermédiaire, est envisageable selon plusieurs dimensions. Si l'on mobilise le concept de dispositif de mobilité, par exemple, dans le cas d'un expatrié - dont la mobilité s'enchâsse dans la première configuration « spatio-temporelle » que nous avons analysé ailleurs (Gherardi et Pierre, 2008) - ce dernier nous apparaît comme un sujet disloqué « là-bas » (au sein d'une filiale étrangère), déconnecté par rapport à « ici » (siège dans le pays d'origine), comme dépourvu de porte parole ou de représentants (au mieux communique-t-il avec un supérieur hiérarchique au siège qui lui demande les résultats de ses actions mais qui ne se préoccupe pas forcément de l'informer des postes disponibles à son retour, des changements de stratégie dans d'autres divisions de l'entreprise, des nominations importantes au siège ou chez les sous-traitants). Cet expatrié occupe une seule scène et a de la peine à maintenir un réseau social, ancré localement, en expatriation. Personne n'est nulle part « à sa place ». C'est le cas également du coordinateur de projets transnationaux (rentrant dans la quatrième configuration (Gherardi et Pierre, 2008) qui se déplace essentiellement pour contrôler les avancées des projets là où ils ont été activés et qui gère des équipes internationales (mais qui ne lui sont pas rattachées hiérarchiquement). Au siège personne n'est en charge de le remplacer ou d'entretenir les contacts pendant son absence. Son capital de liens est faible : il ne peut pas obtenir facilement des informations non redondantes qu'il va pouvoir recouper, contrôler, pour fonder un avantage concurrentiel ; il ne peut pas aisément mettre en relation des personnes qui ne l'auraient jamais été sans lui et faire des «ponts interpersonnels » rapprochant des parties déconnectées de manière productive dans son organisation et en dehors ${ }^{14}$.

Nos enquêtes font état que les moins biens lotis des cadres mobiles quand ils font un bilan et expriment le sens de leur trajectoire professionnelle sur plusieurs années la saisissent aussi souvent comme une réponse à une injonction, un devoir de bouger dicté par l'institution et avec la contrainte bien souvent d'avoir dû se couper de leurs racines en cas d'expatriation longue. Pour ces populations salariées, c'est comme si la conquête sociale, même lorsqu'elle est réussie, correspondait à un état d'enkystement progressif dans un rôle enfin atteint, dans " un cortège de permanences de personnages » qui signe une partie du monde de l'expatriation (Barrère et Martuccelli, 2005). Ils évoquent aussi une prolifération, année après année, des départs «passifs » pour des missions courtes (une à deux journées) "où ils ne prenaient pas réellement de décisions mais étaient là, en réunion, parce qu'il fallait bien être là et représenter le siège, contrôler, parfois faire semblant de contrôler... ». Ici, dans ces témoignages, le déplacement (les occasions de sorties après le travail quand on est à l'étranger, les contacts liés avec des réseaux sur place...) importe peu en dehors de la mission à remplir puisque "le plus tôt était le mieux pour rentrer chez soi! ». Les cadres interrogés disent apporter, dans ces cas, une "faible attention aux destinations qui sont en réalité interchangeables » et disent craindre une dévaluation cumulative de leurs compétences, "un risque d'obsolescence de leurs savoirs à terme. Cela arrive vite et

14 Ronald. S. Burt (2001) observe que les individus qui sont des intermédiaires uniques entre des réseaux cloisonnés connaissent une promotion plus rapide que ceux qui appartiennent à des réseaux en cliques. 
sournoisement». Ces cadres ne peuvent muer en ressources personnelles, investir dans d'autres champs professionnels, ce qu'ils apprennent dans une partie de l'entreprise, ici et maintenant. Leur capital international est tributaire du champ dans lequel il est acquis $^{15}$.

Les mieux lotis des populations étudiées semblent pouvoir utiliser un capital international de différentes manières. Ils circulent mais gardent le contact. Ils font face, mieux que les autres, à des discontinuités socio-temporelles, et à un monde de l'entreprise qui devient plutôt plus grand au fil des ans, puisqu'il est constitué par un réseau contenant davantage de nœuds (souvent mieux interconnectés). Étonnamment, le lieu visité à l'étranger est souvent premier dans le déterminant des choix et ce déplacement (pour une part touristique) importe en dehors de l'objectif professionnel à atteindre. Le lieu visité doit toujours permettre d'actualiser une capacité de mobiliser dans diverses arènes d'emploi des ressources (réseaux professionnels, stocks d'information) utiles ensuite dans la mémoire des situations, la perception des « signaux faibles » quand le temps presse et que l'on doit exprimer un choix sur un projet, une capacité à faire des impasses, être régulier dans ses performances, savoir choisir ses outils face à la situation... et être jugé, au final, comme compétent. Dès lors, ce qui permet la stabilité émotionnelle de la personne est moins la fixité des contenus que la continuité d'un pôle de traitement de l'expérience du monde (Lévy, 2008 : 353), à savoir le fait d'apprendre constamment et de recycler « là-bas » ce qui a été appris « ici », et pouvoir en parler « ici » et « là-bas ». Ces « gagnants » le sont sur deux tableaux, celui de la coexistence spatiale et celui de la relation qui est davantage chronologique et simultanée ${ }^{16}$ et se solidifie au travers d'existences successives (Lévy, 2008 : 127). Dès lors, on comprendra que pour les mieux lotis, ne plus pouvoir bouger est plus que jamais un signe d'impuissance sociale.

Pendant longtemps, pour les expatriés du bas de l'échelle, les différences de statuts et de fonctions étaient contrebalancées par des éléments fédérateurs : relations privilégiées avec les directions d'entreprise, aménagements de carrières, dispositifs

15 Sur un autre terrain d'étude, Anne-Catherine Wagner donne les exemples du bilinguisme des transfrontaliers comme du savoir-faire transnational des routiers ou des cheminots difficilement monnayable en dehors des secteurs professionnels dans lesquels il est accumulé (du fait de la segmentation des réseaux, des règles particulières de la profession). Anne Catherine Wagner propose un intéressant jeu de renforcements croisés pour les hautes classes qui n'ont nul intérêt à renoncer aux fondements nationaux de leur supériorité sociale, mais qui ne peuvent plus non plus se limiter à ces supports. " Ce sont ceux qui possèdent au plus haut niveau la culture dominante de leurs pays qui sont le mieux placés pour donner une forme internationale à leurs capitaux ». "Il n'y a pas, pour les élites, à choisir entre une consécration nationale ou une reconnaissance internationale. Au contraire, le capital international permet de jouer avec différents principes de légitimité, de faire valoir tantôt l'excellence dans le système national, tantôt la valeur des expériences internationales » (2007: 62).

16 Olivier Vilaça (2001 : 191) définit le capital spatial comme l'ensemble des compétences qu'un individu peut mobiliser à chaque fois qu'il est confronté à un problème lié à l'espace. Il regroupe toutes les connaissances dont l'individu dispose pour gérer son rapport à l'espace et signe différentes manières d'habiter un territoire et de donner vie à plusieurs identités. 
statutaires les distinguant des autres cadres (caisses de retraites complémentaires), valorisation du titre associé à la modernité et à l'aventure... Aujourd'hui, nombre de cadres expatriés cessent d'être des relais de pouvoir dans l'organisation. Avec la distance accrue entre cadres « ordinaires » et cadres dirigeants ou d'état-major, ce qui éclate c'est certainement d'abord l'homogénéité par rapport à l'exercice au pouvoir de décision de la population mobile proposée par la vulgate du management international.

\section{Mobilité géographique et exploitation des réseaux sociaux inter- nationaux. La figure des « ubiquistes ».}

\section{Transformer les lieux en espaces}

Wagner constate que la notion de capital, telle qu'elle est mobilisée dans les travaux de Bourdieu, est implicitement liée à des investissements dans des champs nationaux. Or, remarque-t-elle, « on assiste à la constitution d'espaces de pouvoir internationaux (dans le domaine des entreprises, mais aussi de l'enseignement supérieur, du pouvoir) qui produisent leurs effets sur les différents espaces sociaux nationaux ». Notre enquête, mettant en œuvre une correspondance entre territoire géographique et réseau social, confirme ce constat. Distinguant les «branchés », les « connectés », les « retranchés » et les « enclavés » en fonction de la dotation en capital économique et en capital social, Lévy développe la notion de « capital spatial » qui permet d'intégrer le stock d'expériences et les capacités d'agir dans un ensemble en échange d'autres capitaux (symboliques, culturels, économiques, sociaux...). Pour Kaufmann, la mobilité renvoie à la « motilité », capital dont on doit contrôler l'accès et les conséquences, et qui permet, pour celui qui en profite, de se jouer des frictions spatiales et temporelles. La motilité se définit comme "un ensemble de facteurs définissant la potentialité à être mobile dans l'espace, soit, par exemple, les capacités physiques, les aspirations à la sédentarité ou à la mobilité, les systèmes techniques de transport et de télécommunications existants et leur accessibilité, les connaissances acquises, comme le permis de conduire, l'anglais international pour voyager » (Kaufmann, Schuler, Crévoisier et Rossel, 2003 : 26). Ces recherches renouvellent notamment l'analyse des aptitudes des « grands mobiles » à "vivre » des déplacements, plus ou moins « réversibles », selon les contextes familiaux et professionnels, et des formes spécifiques de détention du capital social.

Dans nos enquêtes, ceux qui sont les mieux lotis dans les populations salariées des trois entreprises étudiées, et que nous nommons les ubiquistes, parviennent, plus que les autres, à transformer les lieux professionnels où ils évoluent en espace. Le lieu devient espace lorsque l'on appréhende, dans leurs dimensions temporelles et spatiales, les pratiques qui s'y déroulent et que l'on est capable d'anticiper sur ce qui va s'y dérouler. Pour De Certeau, « est un lieu l'ordre (quel qu'il soit) selon lequel des éléments sont distribués dans des rapports de coexistence. S'y trouve donc exclue la possibilité, pour deux choses, d'être à la même place. La loi du "propre" y règne : les éléments considérés sont les uns à côté des autres, chacun situé en un endroit "propre" et distinct qu'il définit. Un lieu est donc une configuration instantanée de positions. Il implique une indication de stabilité. Il y a espace dès qu'on prend en considération des 
vecteurs de direction, des quantités de vitesse et la variable du temps. L'espace est un croisement de mobiles. Il est en quelque sorte animé par l'ensemble des mouvements qui s'y déploient ${ }^{17}$. Se saisissant de ce constat, Stock et Duhamel proposent un classement des circulations géographiques et des pratiques de mobilité. Ils distinguent cinq critères par rapport à l'espace et au temps : quotidien/non quotidien, choix/ obligation, familier/étranger, proche/loin, non exotique/exotique. La mobilité d'un athlète kenyan qui s'installe pendant trois mois dans un camp d'entraînement américain (non quotidienne, obligée, familière, lointaine, exotique) sera, par exemple, à distinguer de celle d'un consultant britannique qui s'installe, à Singapour, après des étapes dans les principales métropoles mondiales (quotidienne, non familière, lointaine, exotique) » (Lévy, 2008 : 143). De même, Cerdin (2004) répartit les salariés selon trois types : les non mobiles inconditionnels, quelles que soient les circonstances, les mobiles conditionnels, en fonction des caractéristiques de la destination ou du moment où pourrait être vécue la mobilité, et les mobiles inconditionnels, quelles que soient les circonstances.

Notre recherche relativise le couple « choix/obligation » quand la mobilité géographique est une norme sociale pour certaines catégories socioprofessionnelles et que la littérature managériale en fait une mise à l'épreuve obligée et un critère décisif de sélection des futurs cadres dirigeants. Parler de mobilité comme choix sous-entend de ne pas forcément distinguer suffisamment rhétoriques de l'imaginaire « mobilitaire » tel que nous l'avons décrit et réalité. La caractéristique la plus marquante de la mobilité du haut de la hiérarchie sociale vient notamment du fait d'être une contrainte en même temps qu'un facteur de distinction recherché (Gherardi, 2009). La mobilité n'est pas sans « coups cachés » quand, peut-être avant toute chose, elle se décline en pouvoir sur les rythmes d'autrui et sur son propre planning de déplacements. Pour parler de mobilité librement choisie, l'option pour la mobilité, en toutes ses formes, devrait être autant valorisée que celle pour la sédentarité, ce qui n'est jamais le cas, à l'exception des élites économiques constituées par les héritiers de grandes fortunes et des rentiers entrant dans notre échantillon et qui peuvent davantage choisir la mobilité ou l'immobilité tout en gardant le don d'ubiquité. Pour les plus habiles, l'ubiquité est en même temps une stratégie de mobilité (pour le pouvoir et la reconnaissance) et une technique de "résistance » (à une mobilité qui est imposée). Ceci nous évoque l'exemple goffmanien du dompteur qui contraint les tigres à se déplacer, dans la cage, à travers la position qu'il y assume - et non pas à travers les ordres que les félins, contrairement à ce qu'on laisse entendre aux spectateurs, ne comprennent pas. Quand les mobilités sont sous contraintes, et elles le sont toujours, ce n'est pas le sujet qui choisit librement les rythmes, un peu comme si la cage du dompteur tournait sur elle-

17 «Est espace l'effet produit par les opérations qui l'orientent, le circonstancient, le temporalisent et l'amènent à fonctionner en unité polyvalente de programmes conflictuels ou de proximités contractuelles. L'espace serait au lieu ce que devient le mot quand il est parlé, c'est-à-dire quand il est saisi dans l'ambiguïté d'une effectuation, mué en un terme relevant de multiples conventions, posé comme l'acte d'un présent (ou d'un temps), et modifié par les transformations dues à des voisinages successifs. À la différence du lieu, il n'a donc ni l'univocité ni la stabilité d'un "propre". En somme l'espace est un lieu pratiqué » (Michel de Certeau, 1990, cité par Mathis Stock, 2004 : 58). 
même. Cela signe pour nous l'ambiguïté de la mobilité. Si nous élargissons le dispositif de mobilité, nous voyons que celui qui est « ubiquiste » est à son tour un « représentant ». Il représente les actionnaires et c'est essentiellement pour aller à la rencontre des grands actionnaires, fournisseurs, clients... qu'il se déplace - ou qu'il fait déplacer les membres de son équipe en «flottille ». Ce n'est pas donc l'opposition mobile/ immobile, mais plutôt l'opposition mobile/ubiquiste qui indique des asymétries dans le degré de liberté et de subordination entre individus et entre groupes sociaux du haut de l'échelle sociale.

\section{Accumulation des privilèges et différences de positions professionnelles}

En s'appuyant sur la métaphore du réseau, Castells (1999 : 90) parle de « glocapolitains (mi-êtres, mi-flux) », expression qui résume bien la double essence des « nouveaux maîtres » et met en valeur la distinction entre les grands mobiles et les immobiles désavantagés, obligés au local puisque ils ne peuvent pas se déplacer. Mais il ne relève que peu les niveaux de pouvoir parmi les mobiles. En fait, dans une logique binaire du réseau, les inclus font face aux exclus. Ceci nous apparaît réducteur. Notre travail sur les formes de mobilité et sur les différences d'exercice du pouvoir, donne raison, au contraire, à l'intuition de Pinçon et Pinçon-Charlot qui intègre une distinction entre capital «patrimonial » et capital « mondain ». Il est vrai qu' « il se construit sur toute la planète un espace (relativement) séparé aux points connectifs de l'espace des flux : hôtels internationaux dont la décoration (...) est partout identique pour que les heureux élus se sentent partout chez eux et partout à l'écart du monde environnant; salons réservés aux personnalité dans les aérogares pour que celles-ci puissent se tenir à distance du vulgum pecus sur les grandes voix de l'espace des flux (...) ; système d'organisation de voyages, de services de secrétariat et d'accueil permettant au cercle fermé des élites de communier selon les mêmes rites partout dans le monde » (Castells, 1999 : 469). Il est également exact de constater que les relations de la grande bourgeoisie se situent très largement en dehors du fonctionnement managérial de l'entreprise, dans des espaces locaux privés construits à l'échelle mondiale pour les plus riches. Aller loin ou plus loin n'a jamais garanti, pour certains, un dépaysement et l'on peut dire que «la puissance sociale se manifeste justement dans la capacité à retrouver un espace privé et privatif dans différents lieux » (Wagner, 2007 : 103). Si pour les managers et dirigeants issus de l'entreprise, la mobilité physique peut s'appuyer sur des savoirs, une inscription dans des réseaux qui donne un avantage par rapport aux locaux, de l'autre, les grands bourgeois sont au dessus de la légitimité conférée par les règles classiques d'une profession. "La classe dominante, dispose de deux formes de capitaux spécifiques en cela qu'elles sont transmises et donc héritées. Le capital patrimonial et le capital mondain restent à peu près inaccessibles à qui n'en bénéficie pas par la naissance » (Pinçon et Pinçon-Charlot, 2007 : 266). Ce qui est pour nous le plus important, c'est que les rentiers et les héritiers de grandes fortunes ont la liberté de choisir la mobilité ou l'immobilité, de déterminer leur propre rythme alors que ce n'est pas le cas pour les dirigeants salariés, ni même pour les nouveaux patrons qui doivent monter personnellement un capital de relations sociales nationales et internationales. Ajoutons que les dominants, lors des déplacements qui sont les leurs, bénéficient de divers lieux d'ancrage (pendant les vacances, hors du temps de travail, 
par exemple, et la possibilité d'user des résidences des autres) et font montre de ce que nous pourrions nommer une "interspatialité ». Par les rallyes, les cercles et les conseils d'administration, la collusion des élites est une collusion de fait pour la grande bourgeoisie, en dehors du seul fonctionnement managérial de l'entreprise ${ }^{18}$. Pour les rentiers et pour les héritiers qui peuvent davantage choisir leur rythme, étant indépendants de la production, leur mobilité est davantage liée à l'autoréalisation et, de manière plus profonde, exprime le pouvoir qu'ils détiennent dans la détermination de leur temps et espaces de vie. Bauman souligne que le capitalisme financier donne à certains la possibilité de se libérer des contraintes spatiales détachant le pouvoir de la responsabilité, ce qui est une nouveauté historique dans le contexte industriel.

Nos recherches font apparaître au moins quatre populations distinctes : les sédentaires, les populations « mobiles » (cadres et dirigeants mobiles salariés, eux mêmes segmentés en quatre configurations spatio-temporelles), les « mobiles bien situés » (« ubiquistes » parmi les) et les « mobiles bien nés » (aristocratie de l'argent détachée des contingences opérationnelles des cadres et dirigeants mobiles salariés). Ces « mobiles bien nés » sont également caractérisés par ce don d'ubiquité qui fait que leur « ancrage social » est non seulement géographiquement éclaté mais se fonde surtout sur la mobilité et le déplacement physique des autres. Ces « mobiles bien nés », plus que tout autre, profitent de la mobilité de pairs et de subordonnés et confirment en cela l'hypothèse que le capital de mobilité est bien associé au capital social. Ils multiplient le rendement d'un capital (économique ou culturel) par la mobilisation, par procuration, du capital d'un groupe (famille, anciens élèves d'écoles d' « élite », club sélect, noblesse) plus ou moins constitué comme tel et plus ou moins pourvu de capital spatial. Ceci implique une forte capacité chez eux de savoir rapidement construire, mémoriser puis partager, une carte des relations sociales entretenues, pratiquées et donc faciles à réemprunter pour soi et pour les services aux autres de son rang.

Ces considérations invitent, selon nous, à complexifier l'opposition entre l'internationalisme des cadres salariés (mobilisant notamment un capital de mobilité physique qui s'appuie sur des savoirs longuement acquis et une inscription dans des réseaux tel que les anciens d'écoles) et le cosmopolitisme de l'élite économique de vieille extraction (actionnaires et propriétaires) (Wagner, 2007) qui dispose d'une richesse qui est rarement personnelle mais appartient à la famille, et au groupe social, et reste à peu près inaccessible à qui n'en bénéficie pas par la naissance (Pinçon et Pinçon-Charlot, 2007 : 266).

Au total, parler de mobilité géographique pour les puissants, c'est signifier l'augmentation de la part relative d'une pratique devenue ressource, celle du déplacement à l'échelle mondiale, sans pour autant diminuer celle des autres. Pour les puissants, petit groupe d'hommes qui cumulent les positions les plus distinctives, les

18 «La grande bourgeoisie, parce qu'elle est organisée en réseaux qui eux-mêmes se structurent en réseaux de réseaux, met en contacts réels et potentiels l'ensemble des individus qui appartiennent objectivement à la classe » (Pinçon et Pinçon-Charlot, 2007 : 61). 
«biens nés » ou les «biens situés », les mérites s'accumulent ${ }^{19}$ et ils profitent, pour cela, de la mémoire de familiers, de pairs, de chasseurs de têtes, de divers réseaux, de la reconnaissance d'autres milieux qui leur assurent des enracinements dans une "multipositionnalité » recherchée (Boltanski, 1973) parce qu'elle pérennise la puissance.

\title{
Aspects conclusifs. Des manières d'être « mobile » en entreprise
}

\author{
"Ce que le pouvoir impose avant tout, c'est un rythme \\ (de toutes choses : de vie, de temps, de pensée, de discours). » \\ (Barthes, 2002)
}

Pour les populations étudiées, la mobilité géographique reste, au final, un privilège et continue d'être un facteur de différenciation sociale et non de fluidification ou d'égalisation des rapports sociaux. Les formes de mobilité internationale, en entreprise, n'aplatissent pas l'échelon qui sépare cadres intermédiaires, cadres supérieurs et dirigeants. La mobilité géographique est donc un vecteur de distinction entre ces groupes sociaux, dans des multinationales qui se disent, par ailleurs, « horizontales », fonctionnant en réseau, mais qui, en réalité, ne réduisent pas ici écarts de salaires, de pouvoir et de prestige social.

Notre recherche conduit à considérer la mobilité comme un système de rapports de pouvoir, de relations d'entraides et de services rendus, d'emboitements temporels en flottille et non seulement de déplacements linéaires strictement géographiques. Il invite à conclure que l'on peut être pourvu de cet ensemble de ressources de différentes manières et aussi que les transformations du contexte économique de la mobilité internationale, notamment son coût pour l'entreprise, ont commencé à entraîner une diversification des manières de faire profession (capital national ou international) qui traduit aussi bien des «déplacements transversaux » dans l'espace social que des déclassements. Notre analyse a insisté sur une règle de distribution liée pour une large part à la plus ou moins grande capacité de l'individu mobile d'exister sur plusieurs scènes (capital spatial de «l'ubiquiste »).

Dans cet article, nous avons voulu également montrer que l'on peut ne pas se déplacer et être mobile ou mieux, « ubiquiste », non seulement au moyen des technologies, mais aussi au moyen de la mobilité (et également de l'immobilité) d'autres individus qui font partiellement « doublures ». Ces derniers, à leur tour, peuvent être mobiles sans être «ubiquistes », entretenant une forme d'exploitation des « doublures mobiles » par les « ubiquistes ».

19 Ce n'est pas le cas des échelons intermédiaires qui doivent convaincre les décideurs de leur talent et de leur dévouement, avec l'assurance de «futurs exploits ». Cela ne se fait pas d'un coup. Pour eux, «le mérite ne s'accumule pas ; l'employé doit chaque jour reconstruire son statut dans l'entreprise à partir de zéro. Les employés sont réévalués dans chaque nouvelle tâche ou nouveau projet ; c'est leur rendement le plus récent qui détermine leur pointage. Un relâchement momentané de l'effort, un faux pas, et vous pouvez être mis à la porte, quelle que soit la qualité et la longévité de vos états de service »(Bauman, 2003 : 16). 
Être capable de faire face aux enjeux de la mobilité ne reviendrait pas à bouger beaucoup mais à être «ubiquiste », à être présents sur des scènes géographiques éloignées qu'il est aujourd'hui possible d'occuper en même temps. De même, « ceux qui se déplacent le plus vite et le plus loin ne sont pas nécessairement les plus libres » (Kaufmann, 2008 : 100). Les plus « libres » sont ceux qui peuvent choisir la mobilité ou l'immobilité tout en gardant leur pouvoir ; il s'agit notamment de l'élite économique de vieille date (héritiers de grandes fortunes) à laquelle le capitalisme financier donne la possibilité de s'affranchir davantage des contraintes spatiales et temporelles liées à la production.

La notion d'ubiquité, éclairée par celle de dispositif de mobilité, montre que la mobilité géographique considérée de manière quantitative n'est pas un indicateur de pouvoir, c'est-à-dire que l'on ne peut pas considérer que celui qui a le plus de « miles » est le plus puissant. La notion d'ubiquité est utile en ce qu'elle permet de complexifier, d'une part, une théorie de l'exploitation des immobiles par les mobiles au sein de réseaux constitués et alors même, d'autre part, que la métaphore du réseau ne rend pas compte des différences en haut de l'échelle sociale (Degenne et Forsé, 2004). On peut aussi se déplacer sans être mobile. Les temps actuels en entreprise nous invitent donc à explorer davantage les socialités nées de présences connectées et, au-delà de l'opposition entre présent et absent, comment se construisent de fines graduations qui incitent à repenser la relation aussi bien lointaine qu'immédiate dans une perspective stratégique de continuité et de double présence volontairement instituée ${ }^{20}$.

Cet article permet d'entrevoir l'intérêt qu'il y aurait aussi à mieux distinguer entre « classe statut» (autour de critères attribués du « dehors ») et « classe identité » ( « vécue du dedans ») pour certaines populations mobiles. Il ne suffit pas d'être doté de ressources suffisantes ou de se situer dans un même champ pour constituer une classe. Il faut également pour cela effectuer « un travail collectif de construction inséparablement théorique et pratique »d'un collectivisme pratique par lequel, mobilisée pour défendre à la fois ses intérêts, sa cohésion interne et sa pérennité, la classe s'affirme comme telle. C'est, par exemple, par un processus de délégation politique qu'un groupe existe par l'intermédiaire de son porte-parole. Il n'y a pas de syndicats, peu de représentants chez les cadres mobiles qui poursuivent des biens reconnus comme communs.

Dans nos sociétés, on attend toujours plus de nous une aptitude à la mobilité. De déployer des compétences pointues et de disposer d'aptitudes imaginatives pour s'approprier des systèmes techniques et les mettre au profit de projets d'entreprise,

20 Vincent Kaufmann $(2008$ : 100) a su montrer que « de même qu'on peut être très peu mobile et se déplacer beaucoup, on peut à l'inverse se révéler très mobile sans sortir de sa ville, en recherchant systématiquement à explorer l'inconnu et le dépaysement ». Dans ses études, «la motilité devient ressource clé de l'intégration à l'étranger et se réfère donc aux facteurs d'accessibilité (les conditions auxquelles il est possible d'utiliser l'offre au sens large), aux compétences (que nécessite l'usage de cette offre) et à l'appropriation (l'utilisation effective de l'offre pour réaliser ses projets) » (Idem : 31$)$. 
voire de projets personnels. Urry a écrit que les différentes mobilités, par le biais de nos différents sens - voyages imaginaires, transmission des images et de l'information, virtualités et déplacements physiques - sont en train de reconstruire, matériellement, le « social comme société » en « social comme mobilité ». Pour lui, les formes de mobilités repérables dans nos sociétés se substituent au concept de société comme centre souhaitable des sciences sociales. La structure des réseaux, leur taille, leur densité, leur hiérarchisation, est censée faire apparaître la structure sociale. L'enjeu même de la critique sociale change en insistant davantage sur les inégalités d'accès (aux transports par les airs, la mer, le rail, les autoroutes, aux câbles de fibre optique pour le téléphone, la télévision et les ordinateurs...) que sur la dénonciation des inégalités liées aux jeux de la reproduction de positions anciennes (Urry, 2005).

\section{Références bibliographiques}

BARRERE Anne et MARTUCCELLI Danilo (2005) La modernité et l'imaginaire de la mobilité : inflexion contemporaine, Cahiers internationaux de sociologie, $\mathrm{n}^{\circ} 118,2005$, pp. 55-79.

BARTHES Roland (2002) Comment vivre ensemble ?, Cours au Collège de France du 2 février 1977, Paris, Le Seuil.

BAUMAN Zygmunt (2003) L'humanité comme projet, Anthropologie et Sociétés, Volume 27, $\mathrm{n}^{\circ} 3$.

BOLTANSKI Luc (1973) L'espace positionnel. Multiplicité des positions institutionnelles et habitus de classe, Revue française de sociologie, vol. 14, pp. 3-26.

BOLTANSKI Luc (1982) Les cadres. La formation d'un groupe social, Paris, Les éditions de Minuit.

BOLTANSKI Luc et CHIAPELLO Eve (1999) Le nouvel esprit du capitalisme, Paris, Gallimard.

BURT Ronald S (2001) Social Capital : Theory and Research, A. de Gruyter.

CABANTOUS Alain (1995) Les citoyens du large. Les identités maritimes en France du XVII'me au XIX ${ }^{e ̀ m e}$ siècle, Paris, Aubier.

CASTELLS Manuel (1998) L'ère de l'information, Tome 1, Paris, Fayard.

CASTELLS Manuel (1999) L'ère de l' information, Tome 2, Paris, Fayard.

CERDIN Jean-Luc (2004) Les carrières dans un contexte global, Revue Management et avenir, $\mathrm{n}^{\circ} 1$, pp. 155-175.

CHIAPELLO Eve (1998) Artistes vs managers, Paris, Métailié.

CLIFFORD James (1997) Routes, Harvard, Harvard University Press.

COURTY Guillaume (2008) Mobilité professionnelle et identité. Trois retours sur la stéréotypée des routiers, Colloque MSFS 2008.

DEGENNE Alain et FORSE Michel (1994) Les réseaux sociaux, Paris, Armand Colin.

De ROSNAY Joël, Préface à BOUCHEZ Jean-Pierre (2004) Les nouveaux travailleurs du savoir, Paris, Éditions d'organisation.

DIMINESCU Dana (2009) Le migrant connecté. Pour un manifeste épistémologique, Migrations/ Société, vol. $17, \mathrm{n}^{\circ} 102$.

DOYLE Judith et NATHAN Max (2001) The hypermobile must not be allowed to rule roost, The Guardian.

FALCOZ Christophe (2001) Des entreprises aux milles visages. Réponse au commentaire de Loïc CADIN, Gérer et comprendre.

GAZIER Bernard (2003) Tous « sublimes ». Vers un nouveau plein-emploi, Paris, Flammarion. 
GHERARDI Laura (2009) Mobilité ambiguë. Pour une sociologie des classes sociales supérieures dans la société contemporaine, Thèse pour le Doctorat de Sciences Sociales (U.C. Milan - E.H.E.S.S. Paris).

GHERARDI Laura et PIERRE Philippe (2008) Population mobile dans les grandes entreprises : compétences acquises et coûts humains, Revue Européenne des Migrations Internationales, volume $24, n^{\circ} 3$.

HANDY Charles (1989) The Age of Unreason, Harvard Business School Press.

INKSON Kerr \& KHAPOVA Svetlana (2008) International Careers, in Smith P.B., Peterson M.F. et Thomas D.C., The Handbook of Cross-Cultural Management Research, Sage.

IYER Paco (2006) L'homme global, Éditions Hoëbeke.

KAUFMANN Vincent (2008) Les paradoxes de la mobilité, Le savoir suisse, PPUR.

KAUFMANN Vincent et WIDMER Éric (2005) L'acquisition de la motilité au sein des familles État de la question et hypothèses de recherche, Espaces et Sociétés, n 120-121, pp. 199-217.

KAUFMANN Vincent, SCHULER Martin, CREVOISIER Olivier et ROSSEL Pierre (2003) Mobilité et motilité. De l'intention à l'action, Cahiers du LASUR 4, EPFL.

KNAFOU Rémi (2000) Les mobilités touristiques et de loisir et le système globale des mobilités, in Michel Bonnet et Dominique Desjeux, Les territoires de la mobilité, Paris, PUF, pp. 85-94.

LAZAROVA Mila \& TAYLOR Sully (2008) Boundaryless Careers, Social Capital, and Knowledge Management : Implications for Organizational Performance, Journal of Organizational Behavior, $\mathrm{n}^{\circ} 30$, pp. 119-139.

LEVY Jacques (2008) L'invention du monde, Paris, Presses de Sciences-po.

LUSSAULT Michel (2003) Mobilités d'ailleurs. Approches comparatives..., Atelier 3, document de travail interne.

McNULTY Yvonne et THARENOU Phyllis (2004) Expatriate return on investment: A definition and antecedents, International Studies of Management \& Organization, vol. 34, n ${ }^{\circ} 3$, pp. 68-95.

MENGER Pierre-Michel (2002) Portrait de l'artiste en travailleur, Paris, Le Seuil.

MIRALLES Pierre (2007) Le management des talents, Paris, L'Harmattan.

NACHI Mohamed (2006) Introduction à la sociologie pragmatique, Paris, A. Colin.

PIERRE Philippe (2003) Le mouvement perpétuel. Dimension imaginaire de la mondialisation, Passerelles, $\mathrm{n}^{\circ} 26$, Printemps-Eté.

PIERRE Philippe (2003) Mobilité internationale et identités des cadres. Des usages de l'ethnicité dans l'entreprise mondialisée, Paris, Éditions Sides.

PINCON Michel et PINCON-CHARLOT Monique (2006) Grandes fortunes. Dynasties familiales et formes de richesse en France, Paris, Payot.

PINCON Michel et PINCON-CHARLOT Monique (2007) Les ghettos du gotha, Paris, Le Seuil. REICH Robert (1993) L'économie mondialisée, Paris, Dunod.

ROBBINS Stephen P. (2000) Essentials of Organizational Behaviour, Prentice Hall.

SAYAD Abdelmalek (1999) La double absence, Paris, Le Seuil.

STOCK Mathis (2004) L'habiter comme pratique des lieux géographiques, EspacesTemps.net.

TAGUIEFF Pierre-André (2001) Résister au « bougisme ». Démocratie forte contre mondialisation techno-marchande, Paris, Mille et une nuits.

URRY John (2005) Sociologie des mobilités, Paris, A. Colin.

VERTOVEC Steven et COHEN Robin (2002) Conceiving Cosmopolitanism : Theory, Context and Practice, Oxford, Oxford University Press.

VILACA Olivier (2001) L'identité des géographes, Sociologies Pratiques, $\mathrm{n}^{\circ} 5$.

WAGNER Anne-Catherine (1998) Les nouvelles élites de la mondialisation. Une immigration dorée en France, Paris, PUF.

WAGNER Anne-Catherine (2007) Les classes sociales dans la mondialisation, Paris, La Découverte.

ZELINSKI W (1971) The hypothesis of mobility transition, The Geographical Review, Vol. 61, $\mathrm{n}^{\circ} 2$, pp. 219-250. 


\title{
Mobilités géographiques et écarts de pouvoir au sein de trois entreprises mondialisées. Mobiles, immobiles et « ubiquistes »
}

\author{
Laura GHERARDI et Philippe PIERRE
}

Cet article s'attaque à la croyance selon laquelle la mobilité géographique internationale, intense et répétée, contribuerait à rendre, en entreprise, une élite homogène de cadres et dirigeants. Cette contribution souligne, au contraire, un espace social de différences dans lequel le groupe de cadres et dirigeants étudiés n'existe pas en tant que classe sociale unifiée. L'article questionne d'abord les usages idéologiques et profits censés découler de la mobilité des idées et des personnes au fil des projets dans une entreprise en réseau. Il met en évidence des dimensions idéologiques de la centralité croissante de la mobilité géographique dans les rapports de domination au travail. Les rentiers et les héritiers de grandes fortunes ont généralement la liberté de choisir la mobilité ou l'immobilité, de déterminer leur propre rythme de déplacement alors que ce n'est pas le cas pour les dirigeants des entreprises étudiées, ni moins encore pour les managers qui doivent acquérir et mobiliser personnellement un capital de relations sociales nationales et internationales. La distinction dans la population même des « mobiles », entre « ubiquistes » et les autres, illustre le lien entre pouvoir et capacité d'un individu à opérer en des scènes géographiquement éloignées, directement ou par délégation. Cette double présence existe non seulement au moyen des nouvelles technologies mais surtout grâce à la mobilité d'autres individus qui se déplacent simultanément au profit des "ubiquistes ». Cette analyse des ressources et potentiels de mobilité physique géographique amène à souligner des formes peu connues d'exploitation et de domination dans les entreprises mondialisées.

\section{Geographic Mobility and Power Differentials in three Multinationals. Mobile, Immobile and "Ubiquitous" Persons}

\section{Laura GHERARDI and Philippe PIERRE}

This article contests the idea that intense, repetitive international geographic mobility contributes to the formation within a corporation of a homogeneous élite of managers and directors, rather it reveals a social space containing disparities within which the group of managers and directors under study does not exist as a unified social class. First of all the article discusses the ideological uses and advantages of mobility of ideas and of persons, from project to project, within the network organisation, and emphasises the ideological aspects of the growing centrality of geographic mobility in command relationships in the workplace. Unlike the company directors studied and the managers who need to personally acquire and make use of a store of national and international social relationships, the rentiers and heirs to large fortunes have the freedom to choose between mobility and immobility, to determine their own rhythm. The distinction, within the population of "mobile persons", between "ubiquitous persons" and others demonstrates the relationship between power and the capacity of an individual to operate in geographically diverse locations, either directly or through delegates: a double presence is possible for the individual, thanks to new technology but above all to other people who move for his benefit, thereby rendering him "ubiquitous". Such an analysis of resources and of the potential of physical geographic mobility brings little-known forms of exploitation and domination to the fore within the global enterprise. 


\section{Movilidades geográficas y diferenciales de poder en tres empresas multinacionales. Móviles, inmóviles y "ubiquistas"}

\section{Laura GHERARDI y Philippe PIERRE}

Este articulo se ataca a la creencia según la cual la movilidad espacial internacional, intensa y repetida contribuye a crear en una empresa una élite homogénea de ejecutivos y dirigentes. El subraya al contrario la existencia de un espacio social de diferencias. El grupo de ejecutivos y dirigentes no existe como clase social unificada. Los autores discuten el uso ideológico y los beneficios que la movilidad de las personas y de las ideas, se supone, aportan al desarrollo de los proyectos de una empresa funcionando en red. Ponen en valor las dimensiones ideológicas de la creciente movilidad y el papel de esta en las relaciones de dominación en el trabajo. Los rentistas y los herederos de grandes fortunas tienen generalmente toda libertad para elegir entre movilidad y inmovilidad, y para determinar el ritmo de sus movimientos. Esto no es el caso de los dirigentes de las empresas estudiadas aquí, menos aún de los managers que necesitan personalmente adquirir y poner en movimiento a un capital de relaciones sociales nacionales e internacionales. Al interior de la población de los "móviles" se puede oponer a los otros los "ubicuistas", oposición que ilustra el lazo entre poder y capacidad de intervención de estos en escenarios geográficamente distantes, actuando directamente o por delegación. Esta doble presencia no se debe solo al desarrollo de las nuevas tecnologías, sino también a la movilidad de otros individuos que se mueven al beneficio de los "ubiquistas". Este análisis de los recursos y de los potenciales de la movilidad física revela formas poco conocidas de explotación y de dominación en el mundo de las empresas mundializadas. 